\title{
Effect of nutritional supplement on bone marrow-derived mesenchymal stem cells from aplastic anaemia
}

\author{
Shihua $\mathrm{Luo}^{1,2} \dagger$, Yinghai Chen ${ }^{3} \dagger$, Lifen $\mathrm{ZhaO}^{1}$, Xia Qi ${ }^{1}$, Xiaoyan Miao ${ }^{1}$, Huimin Zhou ${ }^{4}{ }^{*}+$ and Li Jia ${ }^{1}{ }^{*} \dagger$ \\ ${ }^{1}$ College of Laboratory Medicine, Dalian Medical University, Dalian 116044, Liaoning Province, People's Republic of China \\ ${ }^{2}$ Department of Traumatology, Shanghai Ruijin Hospital, Jiaotong University, Shanghai 200025, People's Republic of China \\ ${ }^{3}$ Department of Radiation Oncology, the Second Affiliated Hospital of Dalian Medical University, Dalian 116027, Liaoning \\ Province, People's Republic of China \\ ${ }^{4}$ Department of Microbiology, Dalian Medical University, Dalian 116044, Liaoning Province, People's Republic of China
}

(Submitted 13 September 2017 - Final revision received 16 December 2017 - Accepted 24 January 2018)

\section{Abstract}

Aplastic anaemia (AA) is characterised by pancytopenia resulting from a marked reduction in haemopoietic stem cells (HSC). The regulation of haemopoiesis depends on the interaction between HSC and various cells of the bone marrow (BM) microenvironment, including BM-derived mesenchymal stromal cells (BMSC). The purpose of this study was to analyse the biological effect of nutritional supplement (NS), a dietary supplement consisting of thirty-six compounds: amino acids, nucleotides, vitamins and micronutrients on the BMSC of AA rats. The AA rat model was established by irradiating X-ray $(2.5 \mathrm{~Gy})$ and intraperitoneal injections of cyclophosphamide $(35 \mathrm{mg} / \mathrm{kg}$; Sigma) and chloramphenicol (35 mg/kg; Sigma). Then AA rats were fed with NS in a dose-dependent manner $(2266 \cdot 95,1511 \cdot 3,1057 \cdot 91 \mathrm{mg} / \mathrm{kg} \mathrm{d}) \mathrm{by}$ intragastric administration. The effect of NS on the BMSC of AA rats was analysed. As compared with AA rats, NS treatment significantly improved these peripheral blood parameters and stimulated the proliferation of total femoral nucleated cells. NS treatment affected proliferative behaviour of BMSC and suppressed BMSC differentiation to adipocytes. Furthermore, NS treatment of AA rats accelerated osteogenic differentiation of BMSC and enhanced bone mineral density. Co-incubation of HSC with mesenchymal stromal cells and serum from AA rats subjected to high-dose NS markedly improved the yield of CD $34^{+}$cells. Protein microarray analysis revealed that there were eleven differentially expressed proteins in the NS group compared with the AA rat group. The identified specific NS might be implicated in rehabilitation of BMSC in AA rats, suggesting their potential of nutritional support in AA treatment.

Keywords: Aplastic anaemia: Nutritional supplements: Bone marrow-derived mesenchymal stromal cells: Rat models

Aplastic anaemia (AA) is a rare and life-threatening heterogeneous bone marrow (BM) failure disorder characterised by peripheral pancytopenia and marrow hypoplasia ${ }^{(1)}$. Although the pathogenesis of AA has been associated with chemicals, drugs, radiation, infection and immune diseases, the exact mechanisms responsible for $\mathrm{BM}$ failure in $\mathrm{AA}$ are unclear. Recently, the main suggested underlying mechanism in AA is a primary haematopoietic stem cell (HSC) deficiency (HSC defect due to an abnormal balance between HSC death and differentiation), an immune-mediated inhibition of haemopoiesis, a defective BM microenvironment (BMME) or a combination of these conditions ${ }^{(2)}$. Because the depression of haemopoiesis in AA is generally attributed to failure of HSC, it is necessary to explore a new stem cell therapeutic measure.
Mesenchymal stromal cells (MSC), also called mesenchymal stem cells, are multipotent progenitors. They were first isolated from $\mathrm{BM}$ and characterised by the ability to differentiate into adipogenic, chondrogenic and osteogenic lineages ${ }^{(3-5)}$. Importantly, BM-derived mesenchymal stromal cells (BMSC) are an essential component of the BM haematopoietic microenvironment. As the abnormal alteration of MSC was observed in patients with AA, various studies have investigated the association between MSC and the pathogenesis of AA. MSC from AA patients have the typical MSC immunophenotype, aberrant cell morphology ${ }^{(6-8)}$, as well as proliferative defects, lower clonogenicity and increased cell apoptosis ${ }^{(9,10)}$. Jiang et $a l^{(11)}$ also showed that the fibroblast growth factor 1 treatment induces the proliferation of BMSC in patients with AA.

Abbreviations: AA, aplastic anaemia; BM, bone marrow; BMD, bone mineral density; BMSC, bone marrow-derived mesenchymal stromal cells; Epo, erythropoietin; HSC, haemopoietic stem cells; IFN- $\gamma$, interferon $\gamma$; MSC, mesenchymal stromal cells; NGF, nerve growth factor; NS, nutritional supplement; RELM- $\beta$, resistin-like molecule $\beta$; SD, Sprague-Dawley; SNS, serum from AA rat group with treatment of high-dose NS.

* Corresponding authors: L. Jia, email jiali0386@sina.com; H. Zhou, email zhouhm@dmu.edu.cn

$\dagger$ These authors contributed equally to this work. 
Moreover the differentiation capacity of AA-derived BM MSC appeared defective ${ }^{(8,9)}$. Treatment with arsenic trioxide partially reversed the differentiation imbalance. Arsenic trioxide could inhibit adipogenic differentiation, but promote osteogenic differentiation in $\mathrm{AA} \mathrm{BMSC}^{(12)}$. However, the underlying mechanism of the influence of BMSC on AA treatment by nutritional supplement (NS) remains unclear and requires further investigation.

Use of natural health products to prevent, inhibit and reverse diseases is gaining increasing importance. Scientific evidence showed that a nutrient mixture, containing ascorbic acid, lysine, proline and green tea extract inhibited matrix metalloproteinase expression and mediated cell invasion and apoptosis in human fanconi anaemia fibroblast cell lines ${ }^{(13)}$. We previously developed strategies to accelerate the rehabilitation of AA mice using nutrient mixture, such as amino acids, nucleotides, vitamins, folate, soyabean phospholipid, inositol, Fe, $\mathrm{Zn}$ and $\mathrm{Mn}$. This nutrient mixture has exhibited synergistic role in AA mice by promoting peripheral blood cells production, marrow nucleated cells proliferation and mitochondria formation, increasing primitive haematopoietic progenitors as well as repairing liver and spleen damage of $\mathrm{AA}$ mice ${ }^{(14,15)}$. In the present study, we examine the effect of NS (containing various kinds of amino acids, nucleotides, vitamins and metal elements) on BMSC from AA rats, evaluating proliferative behaviour of BMSC, suppressing BMSC differentiation and analysing protein expression.

\section{Methods}

\section{Nutrient mixture}

The nutrient mixture was supplied by Dalian Golden Axe Biological Technology Development Co., Ltd and made of lysine (6.6\%), methionine $(8.8 \%)$, phenylalanine $(7.7 \%)$, threonine $(3.3 \%)$, tryptophan $(2.2 \%)$, arginine $(8.38 \%)$, histidine $(5.5 \%)$, glycine $(3.3 \%)$, aspartic acid (3.3\%), leucine (3.3\%), isoleucine $(3.3 \%)$, valine $(4.4 \%)$, serine $(2.2 \%)$, glutamine $(3.3 \%)$, taurine $(2 \cdot 2 \%)$, whey acid $(3.3 \%)$, nucleotide $(4.4 \%)$, niacin $(0.13 \%)$, folate $(0.0099 \%)$, inositol $(0.66 \%)$, Soyabean phospholipid (13.23\%), vitamin A (0.0066\%), vitamin C (1.1\%), vitamin D (0.000066\%), vitamin $\mathrm{B}_{2}(0.022 \%)$, vitamin $\mathrm{B}_{6}(0.022 \%)$, vitamin $\mathrm{B}_{12}(0.000044 \%), \mathrm{Fe}(0.11 \%), \mathrm{Zn}(0.11 \%), \mathrm{Mn}(0.088 \%)$, $\mathrm{Cu}(0.022 \%), \mathrm{Se}(0.001 \%), \mathrm{Cr}(0.0002 \%), \mathrm{K}(3.3 \%), \mathrm{Ca}(3.3 \%)$, $\mathrm{Mg}(2 \cdot 2 \%)$ (online Supplementary Table S1). According to the proto-prescription, oral application was prepared.

\section{Animals and treatments}

In order to induce an AA model, 6-week-old healthy male Sprague-Dawley (SD) rats ( $n$ 126; mean body weight $191 \cdot 1$ (SD 16.2) g) were obtained from Animal Facility of Dalian Medical University. All of experimental procedures were approved by the Animal Studies Ethics Committee of the Dalian Medical University, China. Rats were housed in animal facility with temperature and humidity setting to $24 \pm 1{ }^{\circ} \mathrm{C}, 55 \pm 10 \%$ and with a $12 \mathrm{~h}$ light $-12 \mathrm{~h}$ dark cycle. In all, $126 \mathrm{SD}$ rats were divided randomly into five groups: control group, AA model group and three AA groups with different dose of the nutrient mixture supplement. The 1st day, AA rat models and the three different doses of the nutrient mixture groups were irradiated by X-ray $(2.5 \mathrm{~Gy})$. Then the rats were treated with intraperitoneal injections of cyclophosphamide $(35 \mathrm{mg} / \mathrm{kg}$, Sigma) and chloramphenicol (35 mg/kg, Sigma) in the $4 \mathrm{th}, 6 \mathrm{th}, 8$ th days. These steps were repeated the 15th day. The control animals were treated with physiological saline. After the 5th day, the rats of nutrient mixture groups were fed with nutrient mixture supplement in a dose-dependent manner (2266.95, 1511.3, $1057.91 \mathrm{mg} / \mathrm{kg} \mathrm{d}$, according to Chinese Medical Dictionary) by intragastric administration. All rats received a standard diet during the experiment. After treatment with NS or physiological saline for 8 weeks, the rats were anaesthetised by isoflurane inhalation and decapitated for following experiments.

\section{Peripheral blood and bone marrow analysis}

The blood samples were collected from the SD rat eye sockets. A $0.5-\mathrm{ml}$ aliquot of blood was anti-coagulated with $1.5 \mathrm{mg} / \mathrm{ml}$ dipotassium EDTA (Sigma). Blood samples were analysed with the Sysmex KX-21N Blood Cell analyser (Sysmex Corporation). Serum was isolated using serum separator tube (Microtainer; Becton Dickinson and Co.). The supernatants were collected for erythropoietin (Epo) and co-culture analysis. Epo levels were determined by an ELISA kit (no. ABIN366328; R\&D Systems). The femoral marrow of SD rats was smeared. The femoral marrow cell suspension in 90\% Ham's F-12K (F-12K; Gibco) and $10 \%$ FBS (Gibco) was used to obtain the total BM nucleated cell count (femoral nucleated cell count (FNCC)) using the basophil channel of the Sysmex KX-21N.

\section{Isolation of bone marrow-derived mesenchymal stromal cells and haemopoietic stem cells}

BMSC and HSC were isolated from SD rats of each group. Rats were gently flushed out of tibias and femurs using F-12K and $10 \%$ FBS. Cells obtained from two tibias and two femurs were plated in $100 \mathrm{~mm}$ culture dish (BD Falcon) in $10 \mathrm{ml}$ of BMSC media at $37^{\circ} \mathrm{C}$ with $5 \% \mathrm{CO}_{2}$. After $24 \mathrm{~h}$, non-adherent cells were collected for purification of $\mathrm{CD}_{3} 4^{+}$HSC. The remaining adherent cells, which were abundant in MSC, were trypsinised and replated until they achieved $>85 \%$ density. Non-adherent cells were incubated with CD34-conjugated magnetic microbeads (Miltenyi Biotec) according to the manufacturer's instructions. Afterwards, $\mathrm{CD}_{3} 4^{+}$populations were isolated by using a separation column placed in a magnetic field, and were then subjected to phenotypic and functional assays with more than $90 \%$ survival rate determined by trypan blue staining.

\section{Phenotypic analysis by flow cytometry}

Surface markers (CD34, CD44, CD45 and CD90) of BMSC were identified using flow cytometric analysis. Approximately $1 \times 10^{5}$ cells (after passage 3) were incubated in the dark for $30 \mathrm{~min}$ at $4^{\circ} \mathrm{C}$ with the fluorescein isothiocyanate (FITC)-conjugated antibodies against CD34 (sc-18917 FITC; Santa Cruz Biotechnology), CD44 (sc-18849 FITC; Santa Cruz Biotechnology), CD45 (no. 554877; BD Pharmingen), CD90 (no. 554894; BD Pharmingen), respectively. After washing with PBS, labelled cells were detected by using a flow cytometer (Beckman Coulter). 


\section{Co-culture assay}

To explore the regulatory role of NS treatment in expansion of HSC, HSC were washed and resuspended in Iscove Modified Dulbecco Medium (Gibco), $50 \mathrm{ng} / \mathrm{ml}$ fetal liver tyrosine kinase-3 ligand, $50 \mathrm{ng}$ / $\mathrm{ml}$ stem cell factor (SCF), $50 \mathrm{ng} / \mathrm{ml}$ thrombopoietin and $20 \mathrm{ng} / \mathrm{ml} \mathrm{IL-3} \mathrm{(all} \mathrm{from} \mathrm{PeproTech).} \mathrm{HSC} \mathrm{were} \mathrm{then} \mathrm{prepared} \mathrm{into}$ suspensions at a concentration of $1 \times 10^{4} / \mathrm{ml}$. Four culture systems comprised this assay: (1) HSC suspensions plus $20 \%$ serum from the healthy control group (HSC+SC), (2) HSC plus $20 \%$ serum from the AA rat group with treatment of high-dose NS (HSC+SNS), (3) HSC plus MSC and SC: HSC suspensions were seeded on a confluent MSC layer with $20 \%$ SC, (4) HSC plus SNS and MSC: HSC suspensions were placed on a confluent MSC layer with $20 \%$ SNS. The cells were incubated at $37^{\circ} \mathrm{C}$ in $5 \% \mathrm{CO}_{2}$ for $7 \mathrm{~d}$. Subsequently the expansion of HSC was analysed by flow cytometry.

\section{Cell proliferation assay}

The suspension of BMSC $\left(1 \times 10^{3} /\right.$ well $)$ were plated in ninety-sixwell plates at a concentration of $100 \mu \mathrm{l}$ of $\mathrm{F}-12 \mathrm{~K}$ medium containing $10 \% \mathrm{FBS}$. The cells were then cultured by incubation at $37^{\circ} \mathrm{C}$ in a $5 \% \mathrm{CO}_{2}$ for $2,4,6,8,10,12$ and $14 \mathrm{~d}$. $10 \mu \mathrm{l} \mathrm{CCK}-8$ (Sigma-Aldrich) solutions were added to the well for $2 \mathrm{~h}$. The absorbance at $450 \mathrm{~nm}$ was immediately measured using a microplate reader. The results were recorded for further comparison.

\section{Colony formation assay}

To measure focus formation, cells $\left(1 \times 10^{3}\right)$ were trypsinised to single-cell suspension and seeded in six-well plates. The cultures were maintained in the F-12K containing $10 \%$ FBS, with medium changes every $3 \mathrm{~d}$, until the appearance of foci from transformed cells was evident. At day 14 , the colonies were stained with $0 \cdot 2 \%$ crystal violet, and foci were counted. Images of the colonies were obtained using a NIKON digital camera.

\section{Osteoblast differentiation and alizarin red staining}

Osteoblast differentiation was performed in cells $\left(2 \times 10^{5} /\right.$ well $)$ plated in twelve-well plates in osteoblast-induction media containing Dulbecco's modified Eagle medium (DMEM) supplemented with $10 \%$ FBS, $10 \mathrm{~mm} \beta$-glycerophosphate, $50 \mu \mathrm{g} / \mathrm{ml}$ L-ascorbic acid-2-phosphate and $10 \mathrm{~nm}$ dexamethasone (SigmaAldrich). The medium was changed every $3 \mathrm{~d}$ during induction period. Ca deposition, at day 14 of osteoblastic differentiation, cells was measured using Alizarin Red staining. Osteoblasts were fixed with $10 \%$ paraformaldehyde for $30 \mathrm{~min}$ at room temperature, washed with PBS, and stained with $0 \cdot 1 \%$ Alizarin Red (Sigma-Aldrich $^{\mathrm{TM}}$ ) for $10 \mathrm{~min}$. Images were captured using a digital camera system (Olympus DP12-2) coupled to an inverted optical microscope (Olympus CKX41; Olympus Optical Co.).

\section{Adipocyte differentiation and oil red $O$ staining}

$4 \times 10^{4} /$ well cells were plated in six-well plates and cultured for $12 \mathrm{~d}$ in adipogenic-induction medium (10\% FBS, $0.5 \mathrm{mmol} / \mathrm{l}$ 1-methyl-3-isobutylxanthine, $1 \mu \mathrm{mol} / 1$ dexamethasone, DMEM supplemented with $10 \mathrm{mg} / \mathrm{l}$ insulin). The media was changed every $3 \mathrm{~d}$. At day 14 of adipocyte induction, cells were rinsed in
PBS, fixed in $10 \%$ formaldehyde, stained in $0.3 \%$ oil red $\mathrm{O}$ solution for $30 \mathrm{~min}$ at room temperature. Lipid accumulations of differentiated cells were then obtained from each experimental group. Images were captured using a digital camera system (Olympus DP12-2) coupled to an inverted optical microscope (Olympus CKX41). The number of adipocytes was counted and adipocyte content was calculated by summing up the number of adipocytes from three representative images.

\section{Bone mineral density analysis}

The bone mineral density (BMD) of the rats was determined using a dual energy X-ray absorptiometry system (Lunar iDXA; GE Healthcare) at the health check centre of the Second Affiliated Hospital of Dalian Medical University. The femurs were scanned at midpoint, and BMD was calculated according to the following formula: BMD $\left(\mathrm{g} / \mathrm{cm}^{2}\right)=$ the bone mineral content $(\mathrm{g} / \mathrm{cm}) /$ bone width $(\mathrm{cm})$.

\section{Real-time PCR analysis}

RNA was extracted using the RNeasy Mini Kit (Qiagen). The first strand complementary DNA was synthesised from $1 \mu \mathrm{g}$ of total RNA using QuantiTect Reverse Transcription Kit (Qiagen). The primers were summarised in the online Supplementary Table S2. Real-time quantitative PCR was performed using SYBRGreen-quantitative real-time PCR Master Mix kit (Toyobo Co.). The data were normalised to the geometric means of the reference gene glyceraldehyde 3-phosphate dehydrogenase (GAPDH). The relative expression levels of each target gene were determined by using Biosystems 7300 Real-Time PCR system (ABI).

\section{Protein microarray}

Total protein was extracted from MSC. The protein concentration was determined by BCA Protein Assay Kit (KC-430; KangChen). Before processing, protein microarray slides specific for rats (ninety proteins, CODE: PAM-G2; RayBiotech) (online Supplementary Table S3) were blocked with blocking buffer for $30 \mathrm{~min}$ and then incubated with samples for $1-2 \mathrm{~h}$ at room temperature. After washing, slides were incubated with diluted biotin-conjugated antibodies at room temperature for $2 \mathrm{~h}$. Then, slides were washed and then reacted with streptavidin-conjugated fluor at room temperature. At last, slides were scanned by Axon scanner. By comparing the signal intensities, relative expression levels of cytokines were made. The intensities of signals were quantified by densitometry. Raw intensities were revised by background and normalised by median. Fold changes in protein expression were calculated.

\section{Western blot analysis}

The results from protein microarray were confirmed by Western blot analysis. Cells were lysed in RIPA buffer (Abcam). Total protein quantification was performed using BCA Protein Assay kit (Pierce) according to the manufacturer's instruction. Equal amounts of the protein $(50 \mu \mathrm{g})$ were resolved by running gel (10\% SDS-PAGE) and blotted onto same size nitrocellulose membranes. After being blocked for $2 \mathrm{~h}$ at room temperature in PBS containing $0 \cdot 1 \%$ Tween 20 (PBST) and 5\% skimmed milk, the membranes were hybridised with the following primary 
antibodies overnight: mouse anti-rat resistin-like molecule $\beta$ (RELM- $\beta$ ) (sc-398922; Santa Cruz Biotechnology), goat anti-rat beta nerve growth factor ( $\beta$-NGF) (AF-556-NA; R\&D Systems), mouse anti-rat IL-2 (sc-133118; Santa Cruz Biotechnology), rabbit anti-rat glial cell line-derived neurotrophic factor receptor $\alpha 2$ (GFR- $\alpha 2$ ) (ab62124; Abcam), rabbit anti-rat interferon $\gamma$ (IFN- $\gamma$ ) (ab7740; Abcam), goat anti-rat SCF (ab101072; Abcam), rabbit anti-rat CXC chemokine ligand 12 (CXCL12) (ab25118; Abcam), mouse anti-rat GAPDH (sc-32233; Santa Cruz Biotechnology), and then incubated for $2 \mathrm{~h}$ with horseradish peroxidaseconjugated secondary antibody (sc-2004, sc-2768, sc-358914; Santa Cruz Biotechnology). The bands were visualised using ECL Western blotting kit (Amersham Biosciences) and were analysed with LabWorks (TM ver4.6, UVP; BioImaging Systems). The level of target protein was normalised to that of GAPDH.

\section{Statistical analysis}

Each experiment was performed at least in triplicate, and the measurements were performed in three independent experiments. Data are expressed as means and standard deviations. The differences between the control and AA groups were evaluated by Student's $t$ test. Comparison of each NS-treated group with the AA group was determined by two-way ANOVA. $P<0.05$ was considered statistically significant. All analyses were performed using SPSS 17.0 statistical packages (SPSS Inc.).

\section{Results}

\section{Nutritional supplement effects on peripheral blood of} aplastic anaemia rats

Peripheral blood parameters were first performed automatically in a haematology analyser. Leucocytes, erythrocytes, $\mathrm{Hb}$ and the mean platelet (Plt) counts were notably lower in the AA rat group than in the control group $(P<0.05$ for all parameters, Table 1$)$. In addition, the mean cell Epo of the AA group was significantly increased to $25 \cdot 62$-fold $v$. that of the control group $(P<0 \cdot 05)$.

After 8 weeks of treatment, peripheral blood parameters of rats from each group were determined. Leucocytes, erythrocytes, $\mathrm{Hb}$ and Plt counts were all increased significantly in the three NS groups compared with the AA rat group, respectively (Table 1). In addition to these increases, there was a significant reduction in Epo at this time $(P<0.05 v$. AA rats). This result showed that NS indeed has effect on peripheral blood parameters and Epo level of AA rats.
Nutritional supplement influences bone marrow of aplastic anaemia rats

To measure the effect of NS on BM of AA rats, the femoral marrow was smeared after NS treatment. The severe damage of $\mathrm{BM}$, characterised by lacking haematopoietic cell islands, was observed in the AA rats. Nutrient mixture-treated mice showed a progressive increase in BM cells after 8 weeks feeding of NS (Fig. 1(a)). In addition, the average number of FNCC isolated from $1 \mathrm{ml}$ of $\mathrm{BM}$ aspirate varied between groups and was significantly lower in AA BM compared with normal BM samples (Fig. 1(a)). There was a significant difference in the number of FNCC isolated from BM aspirates of untreated and treated AA rats with NS. Nutrient mixture of high dose markedly increased all BM cell population of AA rats (Fig. 1(b)). The results also indicated that NS increased the population of BM cells in AA rats.

Nutritional supplement promotes the proliferation of bone marrow-derived mesenchymal stromal cells in aplastic anaemia rats

To determine the potential role of NS affecting proliferation of BMSC, AA rats were treated with NS followed by BMSC isolation from mononuclear cells derived from BM samples. Flow cytometry analysis showed that the BMSC were positive for the specific surface markers CD44 (93.9\%) and CD90 (95.1\%), whereas negative for the haematopoietic markers CD34 (0.56\%) and CD 45 (0.77\%) (online Supplementary Fig. S1). According to the results of the CCK- 8 assay in Fig. 2(a), BMSC of AA rats grew slowly when compared with the control group. Interestingly, BMSC from high-dose NS were in a latent period of growth between days 0 and 2, exhibited logarithmic growth between days 2 and 6, and reached a plateau after day 14. Compared with BMSC of AA rats, the proliferation rate of BMSC of NS groups was increased, respectively. A time-dependent increase in proliferation rate was observed in all experimental groups, indicating the ability to maintain proliferation during long-term culture.

Subsequently, we assessed the colony-forming capacity of BMSC in AA rats after treatment with NS. As shown in Fig. 2(b), the total number of colonies was significantly lower in BMSC of AA rats compared with the control group. To compare the effects on AA rats with treatment of NS, we conducted the same series of analyses based on BMSC-derived colony formation, demonstrating that colony formation was increased by NS treatment in AA rats (Fig. 2(b)). Therefore, it was inferred that NS might promote the proliferation of BMSC in AA rats.

Table 1. Nutritional supplement affects parameters of peripheral blood of rats (Mean values and standard deviations)

\begin{tabular}{|c|c|c|c|c|c|c|c|c|c|c|c|}
\hline \multirow[b]{2}{*}{ Groups } & \multirow[b]{2}{*}{$n$} & \multicolumn{2}{|c|}{$\mathrm{EPO}(\mathrm{mU} / \mathrm{ml})$} & \multicolumn{2}{|c|}{ Leucocytes $\left(\times 10^{9} / \mathrm{I}\right)$} & \multicolumn{2}{|c|}{ Erythrocytes $\left(\times 10^{12} / \mathrm{l}\right)$} & \multicolumn{2}{|c|}{$\mathrm{Hb}(\mathrm{g} / \mathrm{l})$} & \multicolumn{2}{|c|}{ PIt $\left(\times 10^{9} / \mathrm{l}\right)$} \\
\hline & & Mean & SD & Mean & SD & Mean & SD & Mean & SD & Mean & SD \\
\hline Control & 26 & $50 \cdot 62$ & 3.17 & 8.49 & $2 \cdot 70$ & $7 \cdot 85$ & $2 \cdot 23$ & 139.98 & $17 \cdot 69$ & 672.47 & 75.08 \\
\hline AA & 25 & $1296 \cdot 86 \dagger$ & $155 \cdot 78$ & $1.74 \dagger$ & 0.81 & $3.59 \dagger$ & 0.97 & $76.51 \dagger$ & 11.32 & $92 \cdot 70 \dagger$ & $16 \cdot 88$ \\
\hline High dose & 25 & $386 \cdot 05^{\star}$ & $69 \cdot 37$ & $7 \cdot 39^{\star}$ & $1 \cdot 31$ & $6 \cdot 84^{*}$ & 1.73 & $139 \cdot 22^{*}$ & 17.08 & $548 \cdot 37^{*}$ & 69.09 \\
\hline Middle dose & 26 & $473.49^{\star}$ & $85 \cdot 16$ & $4 \cdot 79^{\star}$ & 0.98 & $5 \cdot 56^{\star}$ & $1 \cdot 38$ & $112 \cdot 26^{\star}$ & $13 \cdot 74$ & $364 \cdot 24^{\star}$ & $45 \cdot 89$ \\
\hline Low dose & 24 & $699 \cdot 01^{*}$ & $128 \cdot 58$ & 2.08 & 0.62 & $4 \cdot 17$ & 1.03 & 85.93 & $10 \cdot 19$ & $225 \cdot 72^{*}$ & 31.64 \\
\hline
\end{tabular}

EPO, erythropoietin; PIt, platelet; AA, aplastic anaemia.

${ }^{*} P<0.05$ v. AA group.

$\dagger P<0.05$ v. control group. 

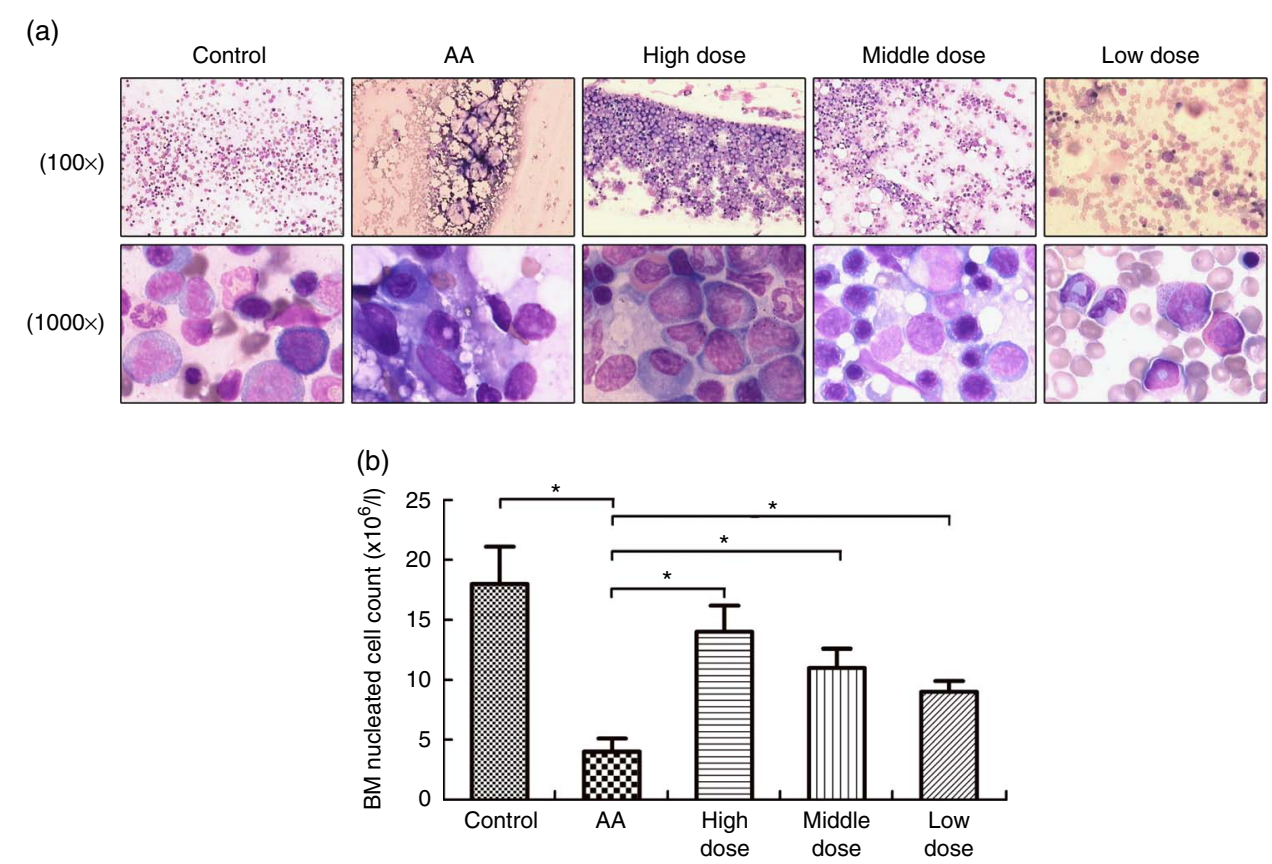

Fig. 1. Nutritional supplement (NS) effects on bone marrow (BM) of aplastic anaemia (AA) rats. (a) After treatment with NS or physiological saline for 8 week, BM smear was performed in femoral marrow generated from control rats, AA rats and NS-treated AA rats with Wright-Giemsa staining, respectively, $100 \times$ or $1000 \times$. (b) The mean BM nucleated cell count was analysed. A decrease of femoral nucleated cell count (FNCC) in the AA group was observed, as compared with the control (* $P<0.05$ ). Significant up-regulation of FNCC was confirmed from NS-treated AA rats compared with the AA group ( ${ }^{\star} P<0.05$ ). Values are means and standard deviations from three independent experiments.

\section{Nutritional supplement promotes the proliferation of}

haemopoietic stem cells by affecting mesenchymal

stromal cells

$\mathrm{CD} 34^{+}$cells were enumerated using flow cytometry analysis over $7 \mathrm{~d}$ of culture. As seen in online Supplementary Fig. S2, there was a significant increase in the amount of $\mathrm{CD} 4^{+}$cells from the HSC + SNS and HSC + MSC + SC groups as compared with control culture. Interestingly, co-incubation of HSC with MSC and SNS markedly improved the yield of CD $34^{+}$cells than other three culture systems, implicating that NS promoted the expansion of HSC possibly by affecting MSC.

\section{Nutritional supplement promotes the differentiation of} bone marrow-derived mesenchymal stromal cells into osteoblasts in aplastic anaemia rats

To address the potential of NS to induce osteoblasts, we investigated differentiation of BMSC into osteoblasts. Formation of osteoblasts was decreased in AA rats compared with the control group (Fig. 3(a)). Furthermore, number of osteoblasts was significantly increased in AA rat treatment with NS compared with the control group, indicating increased osteoblast differentiation in dose-dependent manner of nutrient mixture.

We examined the transcription factors alkaline phosphatase $(A L P)$ and osteocalcin $(B G L A P)$ involved in the osteoblast differentiation at a time interval of $14 \mathrm{~d}$. The $A L P$ and $B G L A P$ mRNA of BMSC was considerably decreased in the AA group compared with the control group (Fig. 3(b)). Furthermore, NS in the AA rats exerted dose-dependent stimulative effect on $A L P$ and BGLAP expression of BMSC during their induction into osteoblast differentiation. These data supported the effect of NS on inducing osteoblast differentiate of BMSC.

\section{Nutritional supplement suppresses differentiation of bone marrow-derived mesenchymal stromal cells into adipocytes in aplastic anaemia rats}

To determine whether NS regulates adipocyte differentiation of BMSC in AA rats, BMSC were induced. We demonstrated that adipocytes of AA rats were significantly increased compared with the control group on day 14 (Fig. 4(a)). In contrast, NS significantly inhibited the commitment of BMSC into adipocyte lineage, suggesting impaired adipocyte differentiation in dose-dependent manner of nutrient mixture (Fig. 4(a)). Thereafter, we analysed the expression of key adipocyte-specific genes during adipocyte differentiation. The mRNA levels of fatty acid-binding protein 4 (FABP4), PPARG, adiponectin and CCAAT/enhancer-binding protein $\alpha(C / E B P \alpha)$ were significantly increased in the AA group compared with the control group (Fig. 4(b)). When BMSC were differentiated into adipocytes, the levels of these genes were significantly diminished in AA rats treated with NS. These data suggested that decreased expression of FABP4, PPARG, adiponectin and $C / E B P \alpha$ by NS suppressed adipocyte differentiation.

\section{Nutritional supplement improves bone mineral density and reduces adipocyte content in aplastic anaemia rats}

Online Supplementary Fig. S3A and 3B show the influence of NS administration on BMD and adipocyte content of AA rats. Compared with the control group, AA rats displayed a decreased BMD of femur, accompanied by an elevation of adipocyte accumulation in MSC culture. As expected, the reduction in BMD was significantly improved and adipogenesis was inhibited in the group exposed to NS at high-dose. BMD of rat groups treated by middle and low dose was only slightly higher than that of the AA group, whereas the adipocyte 


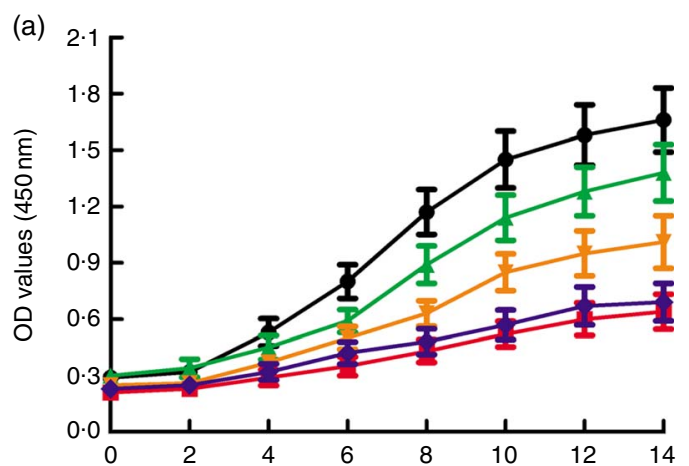

(d)
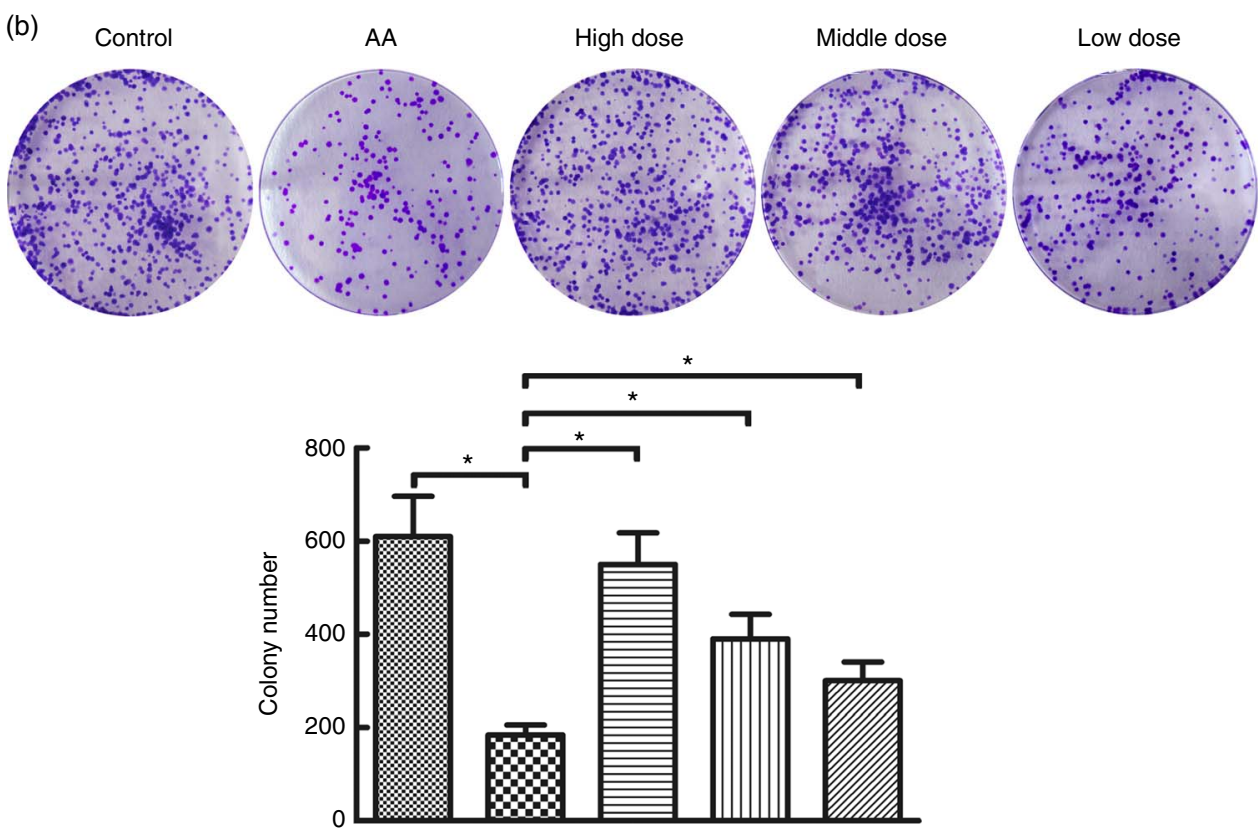

Fig. 2. Nutritional supplement (NS) effects on proliferation of bone marrow-derived mesenchymal stromal cells (BMSC) in aplastic anaemia (AA) rats. (a) Growth curves of BMSC in AA rats were compared with control, and NS-treated groups compared with the AA group with the CCK-8 assay. OD, optical density. (b) The colony-forming activities of BMSC were performed from control, AA rats and NS-treated AA rats. Morphology foci were observed at the microscope and photographed. Significant regulation of colony formation numbers was confirmed in BMSC from AA rats compared with control, and NS-treated groups compared with the AA group $\left({ }^{*} P<0.05\right)$. Values are means and

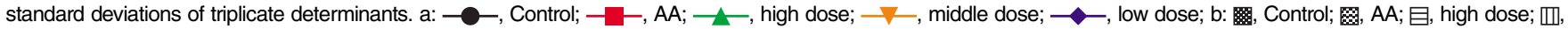
middle dose; 四, low dose.

content of AA rats lowered obviously in response to NS treatment at middle and low dose.

\section{Nutritional supplement mediates protein expression of} bone marrow-derived mesenchymal stromal cells in aplastic anaemia rats

To assess the validity of NS against proteins expressed in AA rats, the BMSC microarrays were first used to compare the protein expression, which was measured on triplicate arrays. Triple protein combinations dominated the candidates identified. Of these ninety differentially reactive proteins, seventeen were highly expressed in BMSC when compared with AA groups for healthy controls. RELM- $\beta, \beta$-NGF, IL-2, GFR- $\alpha 2$ and IFN- $\gamma$ were all significantly higher in the AA group than the control group (Fig. 5(a)). In comparison to AA groups, eleven proteins were differentially reactive for the AA group treated with NS, respectively. Interestingly, RELM- $\beta, \beta$-NGF, IL-2, GFR$\alpha 2$ and IFN- $\gamma$ levels were inhibited in BMSC of AA rats treated with NS (Fig. 5(b)). The levels of these five molecules were further confirmed by Western blot analysis. Moreover, prohaematopoietic cytokines, SCF and CXCL12, showed declined expression in AA rats compared with control and enhanced levels in response to high-dose NS treatment as determined by Western blotting (online Supplementary Fig. S4). Our results indicated that NS addition was able to mediate the protein expression of BMSC in AA rats.

\section{Discussion}

In the present study, to help understand the possible involvement of NS in the reduced haemopoiesis observed in the BM of AA rats, the basic properties of BMSC from AA rats were compared with those derived from AA rats samples treated with NS. Several 
(a)
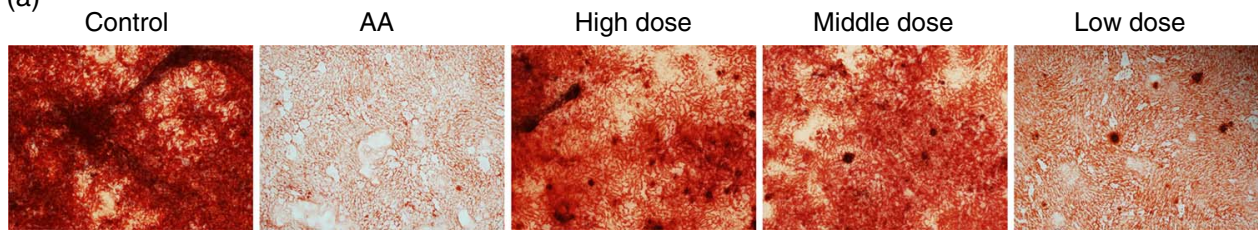

(b)

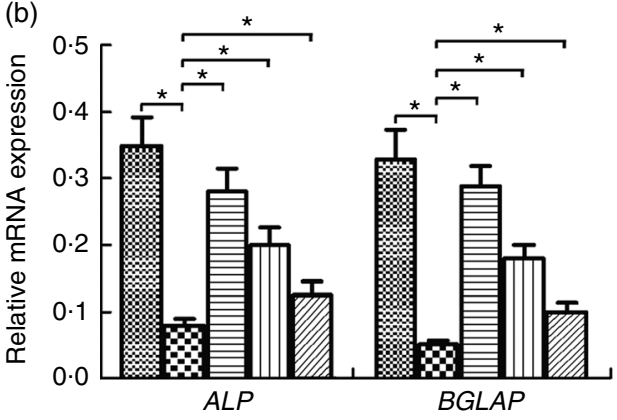

Fig. 3. Effect of nutritional supplement (NS) on the differentiation of bone marrow-derived mesenchymal stromal cells (BMSC) into osteoblasts in aplastic anaemia (AA) rats. (a) Alizarine Red staining was performed after $14 \mathrm{~d}$ of NS treatment. (b) quantitative PCR analysis of osteoblastic gene expression in BMSC induced to osteoblast differentiation by NS treatment. The expression of each target gene relative to that of glyceraldehyde 3-phosphate dehydrogenase (GAPDH) was calculated $\left({ }^{\star} P<0.05\right)$. Values are means and standard deviations of three independent experiments. b: 図, Control; 圈, AA; 目, high dose; 四, middle dose; 四, low dose; ALP, alkaline phosphatase; BGLAP, osteocalcin.

(a)

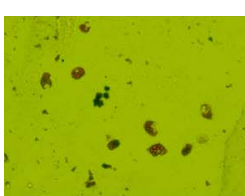

AA

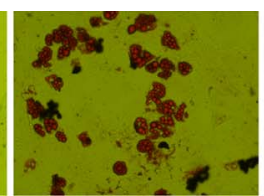

High dose

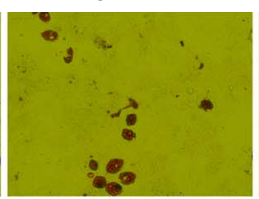

Middle dose

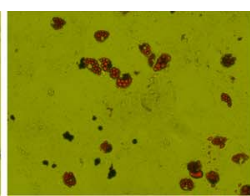

Low dose

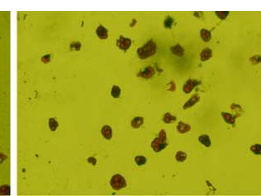

(b)

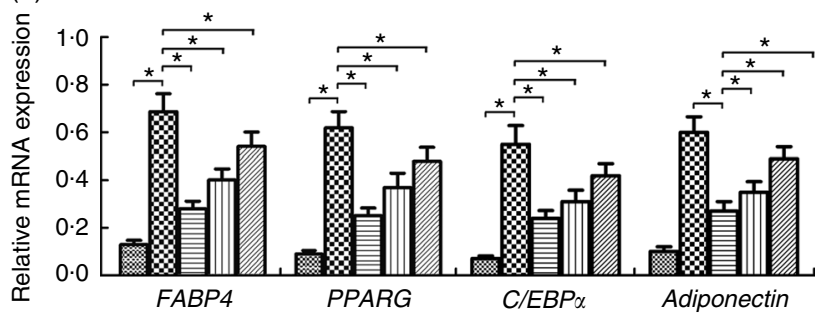

Fig. 4. Effect of nutritional supplement (NS) on the differentiation of bone marrow-derived mesenchymal stromal cells (BMSC) into adipocytes in aplastic anaemia (AA) rats. (a) Oil Red O staining and the area of mature adipocytes were shown after $14 \mathrm{~d}$ of NS treatment. (b) The adipocyte-specific gene fatty acid-binding protein 4 (FABP4), PPARG, adiponectin and CCAAT/enhancer-binding protein a $(C / E B P a)$, were analysed by quantitative real-time PCR. The expression of each target gene relative to that of glyceraldehyde 3-phosphate dehydrogenase $(G A P D H)$ was calculated $\left({ }^{\star} P<0.05\right)$. Values are means $(n 3)$ and standard deviations and representative of three independent BMSC lines. b: 図, Control; 圈, AA; 目, high dose; 四, middle dose; 四, low dose.

differences were identified. Our observations might be explained, at least in part, by a potential nutritional support in AA treatment.

$\mathrm{AA}$ is a rare disease in which the normal generation of erythrocytes and various white cell lines in the BM is disrupted $^{(16)}$. In this work, we developed an animal model of AA, by irradiation with X-ray and treatment with intraperitoneal injections of cyclophosphamide and chloramphenicol in rats. In order to confirm that we were in fact in the presence of an animal model of this haematological disorder, we assessed the parameters. As compared with the control group, AA rats showed significant reduction in peripheral blood leucocytes, erythrocytes, $\mathrm{Hb}$ and plt, even more severe decrease in femoral nucleated cell isolated BM aspirate, and increase in serum Epo, suggesting that we achieved X-ray, cyclophosphamide and chloramphenicol-mediated AA rat model.

Although numerous studies have documented their association with AA, investigations involving the role of NS in AA treatment are considered to be limited. Indeed, Jaako et al. ${ }^{(17)}$ reported that amino acid L-leucine treatment significantly improved the number of erythrocytes, $\mathrm{Hb}$ concentration, $\mathrm{BM}$ cellularity and alleviated stress haematopoiesis in Rps19-deficient anaemia mice. Payne et al. ${ }^{(18)}$ demonstrated in their study that L-leucine can activate the mTORC1 signalling pathway and act as useful therapeutic agent for Diamond-Blackfan anaemia 
(a)

$$
\begin{aligned}
& \text { Colour key } \\
& \begin{array}{ll}
-1 & 1
\end{array}
\end{aligned}
$$

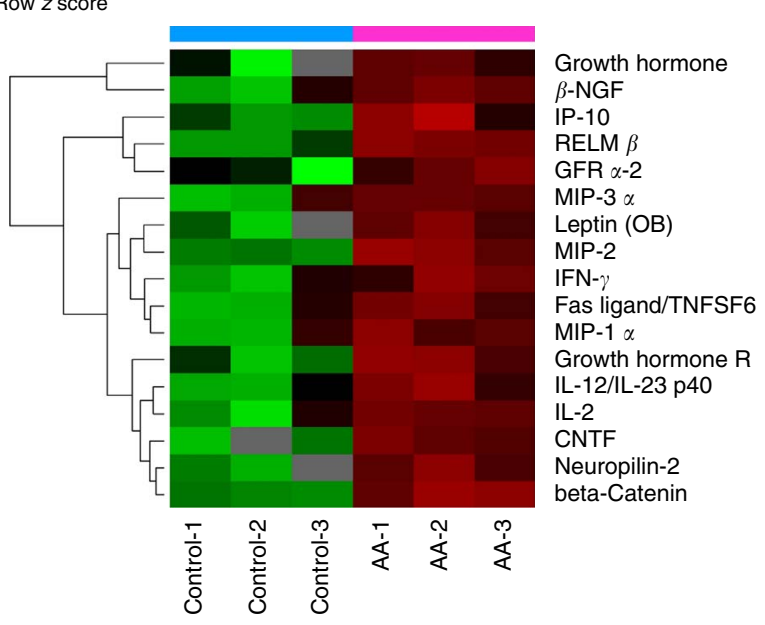

\section{Name}

RELM $\beta$

beta-Catenin

MIP-2

CNTF

Growth hormone R

beta-NGF

Neuropilin-2

Leptin (OB)

IL-2

IL-12/IL-23 p40

IP-10

Fas ligand/TNFS F6

GFR $\alpha-2$

\begin{tabular}{|c|c|}
\hline$A A$ & \\
\hline Control & $P$ \\
\hline $2 \cdot 8856278$ & 0.0002865 \\
\hline 3.5822138 & 0.0006408 \\
\hline 5.809615 & 0.0030692 \\
\hline 4.031222 & 0.0055946 \\
\hline $5 \cdot 1232191$ & 0.0071886 \\
\hline 7.0732539 & 0.0104354 \\
\hline 3.9862081 & 0.0123029 \\
\hline $6 \cdot 3502964$ & 0.017996 \\
\hline 2.5771409 & 0.0180377 \\
\hline 2.9963211 & 0.0213578 \\
\hline 3.5588746 & 0.0229441 \\
\hline 3.1253313 & 0.0278556 \\
\hline $2 \cdot 189021$ & 0.0309115 \\
\hline $2 \cdot 3244991$ & 0.0417713 \\
\hline $2 \cdot 6926164$ & 0.0463427 \\
\hline 3.04885 & 0.0463976 \\
\hline 7.5723701 & 0.046715 \\
\hline
\end{tabular}

IFN- $\gamma$,

MIP- $1 \alpha$

MIP-3 $\alpha$

Growth hormone
High dose

Name
RELM $\beta$
RALT/MIG-6
IL-2
TIMP-1
GFR $\alpha-2$
Fractalkine
Follostatin-like -1(FSL1)
TGF- $\beta 1$
IFN- $\gamma$
VEGF
$\beta$-NGF

\begin{tabular}{ccc}
\hline AA & & $P$ \\
0.2109161 & & 0.0002585 \\
0.2791785 & & 0.0007328 \\
0.3398211 & & 0.0008536 \\
0.2013133 & & 0.0034751 \\
0.3183232 & & 0.0039099 \\
0.1064227 & & 0.0047297 \\
0.1893172 & & 0.0129855 \\
0.2957133 & & 0.0139615 \\
0.3394864 & & 0.0192934 \\
0.1784808 & & 0.0229552 \\
0.2832501 & & 0.0365418
\end{tabular}

$\beta$-NGF

0.2832501

(b)

$$
\begin{aligned}
& \text { Colour key }
\end{aligned}
$$

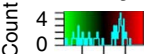

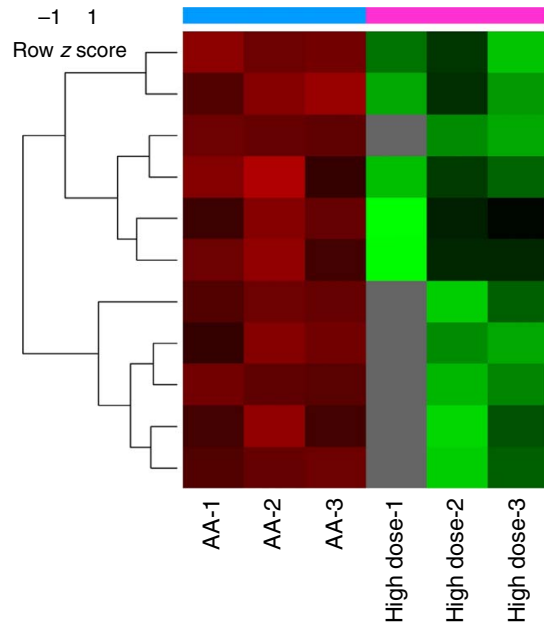

RALT/MIG-6

GFR $\alpha-2$

RELM $\beta$

VEGF

IFN- $\gamma$

Follostatin-like-1(FSL1)

Fractalkine

TGF- $\beta 1$

IL-2

beta-NGF

TIMP-1

Fig. 5. Nutritional supplement (NS) mediates protein expression of bone marrow-derived mesenchymal stromal cells (BMSC) in aplastic anaemia (AA) rats. Protein microarray containing ninety expressions confirmed proteins were probed with BMSC, as described in the 'Methods' section. (a) The image of protein microarray was tested by the control and AA groups. Significant increases in the seventeen proteins of BMSC recognised from AA groups to controls were indicated. MIP, macrophage inflammation protein; CNTF, ciliary neurotrophic factor. (b) The image of protein microarray was tested by AA and NS-treated groups. Of these ninety differentially reactive proteins, eleven were lowly expressed in BMSC when compared with NS-treated groups for AA groups. Values are means and standard deviations of three independent experiments. RELM- $\beta$, resistin-like molecule $\beta$; GFR- $\alpha 2$, glial cell line-derived neurotrophic factor receptor $a 2$; IFN- $\gamma$, interferon $\gamma$.

$(\mathrm{DBA})^{(18)}$. Yu et al. ${ }^{(19)}$ showed that the appropriate vitamin D supplementation could partly correct the immune dysfunction by strengthening signal transduction through vitamin $\mathrm{D}$ receptor in patients with AA. DBA was also associated with higher serum levels of vitamin $\mathrm{B}_{12}$ and folate, which would provide an efficient method for ruling out these deficiencies in the investigation of chronic anaemia ${ }^{(20)}$. Given that a single or a complex of several micronutrients supplementation is helpful in the recuperation process of AA, we prepared NS according to a novel formula of nutrient mixture with thirty-six ingredients, including different amino acids, nucleotide, vitamin, folate, soyabean phospholipid, inositol, iron, zinc, manganese, etc., which was added in the daily diet of AA rats, subsequently the effect and underlying mechanism were explored. After 8 weeks of NS administration, AA rats showed a reduction in erythrocytes, leucocytes, Plt numbers and the $\mathrm{Hb}$ concentration, and these parameters were significantly improved on NS treatment. Whereas Epo was reduced in AA rats treated with NS. In addition, NS had effect on BM nucleated cell recovery in AA rats. The hypocellular blood and BM in AA rats were significantly improved on NS treatment, providing an excellent approach to test novel therapeutic agents enhancing AA rat haematopoiesis. 
AA patients have a hypocellular BM which is replaced by abnormal BM, likely of mesenchymal origin, further supporting a potential contribution of the BM microenvironment to the pathogenesis of $\mathrm{AA}^{(8)}$. It has been shown in several studies that BMSC played a vital role in modulating BM microenvironment and supporting haematopoiesis ${ }^{(21)}$. Graft $v$. host disease is a complication commonly occurred in patients with haploidentical haematopoietic stem-cell transplantation. However, clinical practice revealed that co-transplantation of MSC can sustain haematopoietic engraftment and decrease the risk of graft failure $^{(22)}$. Moreover, MSC in AA patients exhibited significantly reduced haematopoiesis-supporting capacity ${ }^{(23)}$. We, therefore, hypothesised that BMSC might contribute, directly or indirectly, to the pathogenesis of $\mathrm{AA}$. In the present study, our data indicated that BMSC from AA rats had impaired functional properties in proliferation and colony-forming capacity. As expected, NS rescued the inhibitory effect of BMSC growth in $\mathrm{AA}$ rats, suggesting that proliferative behaviour of BMSC was mediated at least in part by NS treatment. Furthermore, a co-culture assay revealed that co-incubation of HSC with MSC and SNS markedly improved the proliferation of $\mathrm{CD} 34^{+}$cells than the HSC + SNS or HSC + MSC groups, implicating that NS promoted the expansion of HSC possibly by affecting MSC.

In general, MSC possess the capacity to differentiate into various types of cells, repair tissue, modulate immune response and support haematopoiesis. MSC have been identified for their ability to differentiate into the bone, adipocytes and cartilage ${ }^{(24)}$. And in some specific microenvironment, for example, injury, MSC could differentiate into the lung epithelial cells in lung injury ${ }^{(25,26)}$. Therefore, efforts to identify the regulatory mechanisms that control the differentiation of BMSC into adipocytes or osteoblasts may lead to the development of new clinical applications and enhance our understanding of haematopoiesis, as BMSC are the primary sources of the haematopoietic microenvironment ${ }^{(27)}$. Although NS play an important role in regulating proliferative behaviour of BMSC, its role in regulating BMSC differentiation of AA rats is unknown. In this study, research was initiated with the analysis of the association between NS and BMSC differentiation. The NS administration accelerated osteoblast, inhibited adipocyte differentiation and reduced accumulation of adipocytes in the AA group. Being a crucial component of haematopoietic niche, the osteoblastic niche has been known for its regulating role in HSC fate $^{(28)}$. A co-culture system of MSC and differentiated osteoblasts could apparently improve the self-renewal capacity and ex vivo expansion of haematopoietic stem and progenitor cell (HSPC) ${ }^{(29)}$. Conversely, marrow adipocytes inhibit the osteoblast differentiation of MSC by suppressing BMPs signalling ${ }^{(30)}$. Our data gave the proof that NS was potential regulator of BMSC differentiation and therefore mediate haematopoiesis. In addition, NS exposure at high dose could significantly enhance BMD in AA rats, implicating the potential roles of NS in alleviating the damage of bone caused by AA.

As a major constituent of BM microenvironment, MSC can reconstitute the damaged stroma and secrete an array of haematopoietic cytokines, stem cell factor and extracellular matrix molecules to promote self-renew and differentiation ${ }^{(21,31)}$. In this study, we used protein microarrays to profile protein expression in lysates obtained from BMSC. A total of ninety derivatives from MSC have been scanned for comparison and analysis. We compared protein profiles of BMSC in AA rats based on whether or not they had received NS treatment during the 8-week study period. However, MSC from the AA group with NS treatment secreted a diverse host of cytokines and haematopoietic growth factors at similar levels compared with those of the AA group, including differentiation and maturationrelated molecules granulocyte colony-stimulating factor, macrophage-CSF, IL-1, IL-3, IL-6, as well as IL-12. The obvious dissimilarity of these factors was not observed between the AA group with or without NS administration. MSC from two groups also exhibited similar expression patterns of anti-inflammatory cytokines IL-10 and 13, adhesion molecules ICAM-1, HSPC homing promoter E-selectin and chemokines such as CCR4, CXCR4. Interestingly, of these ninety differentially reactive proteins, seventeen proteins were highly expressed in BMSC from the AA group when compared with the healthy control group, whereas eleven proteins had low levels in the AA group treated with NS compared with the AA group. Most of these altered factors are effective regulators for regenerative medicine. For instance, RELM- $\beta$ belongs to a family of cysteine-rich secretory molecules initially described to control insulin resistance in rodents. RELM proteins have been shown to modulate inflammation and wound healing processes ${ }^{(32)}$. Zanotti et al. ${ }^{(33)}$ show that MSC are able to suppress activation of endothelial cells, angiogenesis and T-cell homing by releasing metalloproteinase-1, thereby exerting anti-inflammation effects. Other inflammation and immunoregulation-associated cytokines such as vascular endothelial growth factor $(\mathrm{VEGF})^{(34)}, \mathrm{TGF}-\beta 1^{(35)}$ and its induced product, follistatin-like protein- $1^{(36,37)}$, were also changed following NS treatment in our study, which accounting for at least partially the regeneration-promoting potency of BM MSC induced by NS. In addition, MSC could be a source of regulators for signalling pathways and cellular interactions. In mouse skin, receptor associated late transducer (RALT) protein behaves as an inhibitor of epidermal growth factor receptor signalling ${ }^{(38)}$. Two molecules, fractalkine (CX3CL1) and VEGF, have been shown to be involved in the process of homing and mobilisation of $\mathrm{HSPC}^{(39)}$. Interleukins play important roles in immune regulation and contribution to haematopoiesis. MSC themselves can produce therapeutic cytokines, such as IL-2 ${ }^{(40)}$. IL-2 has been reported to be increased in acquired $\mathrm{AA}^{(41)}$, that was consistent with our findings. NGF plays an important role in the nervous system by supporting the survival and growth of neural cells, regulating cell growth, promoting differentiation into neuron, and neuron migration. $\beta$-NGF gene transfection promotes differentiation of BMSC into neurons through regulation of AKT and MAPK pathway ${ }^{(42)}$. GFR $\alpha 2$ is described as a pituitary stem/progenitor cell marker involved in central and peripheral nervous system development ${ }^{(43,44)}$. MSC from AA patients had deficient immune suppression of IFN- $\gamma$ release ${ }^{(6)}$. Increased production of IFN $-\gamma$ has been confirmed to contribute to the pathogenesis of acquired $\mathrm{AA}$, and immunosuppressive therapy may induce haematological remission by suppressing IFN- $\gamma^{(45)}$. We observed that above bioactive compounds released by MSC differed significantly between groups. With NS treatment at high dose, MSC also pronounced increased levels 
of HSC maintenance factors, including SCF and CXCL12. Thus, nutritional treatment may realise their ability to facilitate the recovery of $\mathrm{BM}$ of $\mathrm{AA}$ rats by regulating the production of cytokines from MSC.

In conclusion, our findings support the hypothesis that NS plays an important role in regulating the proliferation and differentiation potential of BMSC and contributes to haematopoietic supporting capacity in AA rats. Furthermore, in BM of AA rats, NS is involved in the protein expression of MHSC, which regulates the haematopoietic microenvironment. Identifying the regulatory mechanism of NS in BMSC may lead to the development of novel therapeutic approaches for AA.

\section{Acknowledgements}

The authors would like to thank Wenguo Wu and Peiman Yang for their help in study design and technical support. The authors would also like to thank Dalian Jinfu Biological Technology Development Co., Ltd for providing the compound design and assistance with trial foods preparations.

This work was supported by funding from the Dalian Transverse Project (project code 20150625).

L. J. and H. Z. designed the study and prepared the manuscript; S. L., Y. C. and L. Z. conducted the research; X. Q. and X. M. analysed the data. All authors read and approved the final manuscript.

None of the authors has any conflicts of interest to declare.

\section{Supplementary material}

For supplementary material/s referred to in this article, please visit https://doi.org/10.1017/S0007114518000399

\section{References}

1. Marsh JC, Ball SE, Cavenagh J, et al. (2009) Guidelines for the diagnosis and management of aplastic anaemia. $\mathrm{Br} J$ Haematol 147, 43-70.

2. Chatterjee S, Dutta RK, Basak P, et al. (2010) Alteration in marrow stromal microenvironment and apoptosis mechanisms involved in aplastic anemia: an animal model to study the possible disease pathology. Stem Cells Int 2010, 932354.

3. Caplan AI (1991) Mesenchymal stem cells. J Orthop Res 9, 641-650.

4. Haynesworth SE, Goshima J, Goldberg VM, et al. (1992) Characterization of cells with osteogenic potential from human marrow. Bone 13, 81-88.

5. Haynesworth SE, Baber MA \& Caplan AI (1992) Cell surface antigens on human marrow-derived mesenchymal cells are detected by monoclonal antibodies. Bone 13, 69-80.

6. Bacigalupo A, Valle M, Podestà M, et al. (2005) T-cell suppression mediated by mesenchymal stem cells is deficient in patients with severe aplastic anemia. Exp Hematol 33, 819-827.

7. Xu Y, Takahashi Y, Wang Y, et al. (2009) Downregulation of GATA-2 and overexpression of adipogenic gene-PPAR $\gamma$ in mesenchymal stem cells from patients with aplastic anemia. Exp Hematol 37, 1393-1399.

8. Li J, Yang S, Lu S, et al. (2012) Differential gene expression profile associated with the abnormality of bone marrow mesenchymal stem cells in aplastic anemia. PLOS ONE 7, e47764.

9. Chao Y, Peng C, Harn H, et al. (2010) Poor potential of proliferation and differentiation in bone marrow mesenchymal stem cells derived from children with severe aplastic anemia. Ann Hematol 89, 715-723.

10. Shipounova IN, Petrova TV, Svinareva DA, et al. (2009) Alterations in hematopoietic microenvironment in patients with aplastic anemia. Clin Transl Sci 2, 67-74.

11. Jiang S, Xia M, Yang J, et al. (2015) Novel insights into a treatment for aplastic anemia based on the advanced proliferation of bone marrow-derived mesenchymal stem cells induced by fbroblast growth factor 1. Mol Med Rep 12, $7877-7882$

12. Zhao J, Wang C, Song Y, et al. (2014) Arsenic trioxide and microRNA-204 display contrary effects on regulating adipogenic and osteogenic differentiation of mesenchymal stem cells in aplastic anemia. Acta Biochim Biophys Sin (Shanghai) 46, 885-893.

13. Roomi MW, Roomi NW, Bhanap B, et al. (2013) Repression of matrix metalloproteinases and inhibition of cell invasion by a nutrient mixture, containing ascorbic acid, lysine, proline, and green tea extract on human Fanconi anemia fibroblast cell lines. Exp Oncol 35, 20-24.

14. Jia L, Yu J, He L, et al. (2011) Nutritional support in the treatment of aplastic anemia. Nutrition 27, 1194-1201.

15. He L, Miao X, Lv G, et al. (2011) Nutritional rehabilitation of mitochondrial aberrations in aplastic anaemia. Br J Nutr $\mathbf{1 0 5}$, 1180-1187.

16. Self SG, Longton G, Kopecky KJ, et al. (1991) On estimating HLA/disease association with application to a study of aplastic anemia. Biometrics 47, 53-61.

17. Jaako P, Debnath S, Olsson K, et al. (2012) Dietary L-leucine improves the anemia in a mouse model for DiamondBlackfan anemia. Blood 120, 2225-2228.

18. Payne EM, Virgilio M, Narla A, et al. (2012) L-Leucine improves the anemia and developmental defects associated with Diamond-Blackfan anemia and $\operatorname{del}(5 q)$ MDS by activating the mTOR pathway. Blood 120, 2214-2224.

19. Yu W, Ge M, Lu S, et al. (2016) Decreased expression of vitamin $\mathrm{D}$ receptor may contribute to the hyperimmune status of patients with acquired aplastic anemia. Eur J Haematol 96 , 507-516.

20. Gelbart D (2014) Diamond-Blackfan anemia and nutritional deficiency-induced anemia in children. JAAPA 27, 36-44.

21. Dazzi F, Ramasamy R, Glennie S, et al. (2006) The role of mesenchymal stem cells in haemopoiesis. Blood Rev 20, 161-171.

22. Ball LM, Bernardo ME, Roelofs H, et al. (2007) Cotransplantation of ex vivo expanded mesenchymal stem cells accelerates lymphocyte recovery and may reduce the risk of graft failure in haploidentical hematopoietic stem-cell transplantation. Blood 110, 2764-2767.

23. Hamzic E, Whiting K, Gordon Smith E, et al. (2015) Characterization of bone marrow mesenchymal stromal cells in aplastic anaemia. Br J Haematol 169, 804-813.

24. Dominici M, Le Blanc K, Mueller I, et al. (2006) Minimal criteria for defining multipotent mesenchymal stromal cells. The International Society for Cellular Therapy position statement. Cytotherapy $\mathbf{8}, 315-317$.

25. Sueblinvong V, Loi R, Eisenhauer PL, et al. (2008) Derivation of lung epithelium from human cord blood-derived mesenchymal stem cells. Am J Respir Crit Care Med 177, 701-711.

26. Wong AP, Dutly AE, Sacher A, et al. (2007) Targeted cell replacement with bone marrow cells for airway epithelial 
regeneration. Am J Physiol Lung Cell Mol Physiol 293 L740-L752.

27. Frenette PS, Pinho S, Lucas D, et al. (2013) Mesenchymal stem cell: keystone of the hematopoietic stem cell niche and a stepping-stone for regenerative medicine. Annu Rev Immunol 31, 285-316.

28. Arai F \& Suda $\mathrm{T}$ (2007) Maintenance of quiescent hematopoietic stem cells in the osteoblastic niche. Ann $N Y$ Acad Sci 1106, 41-53.

29. Huang $\mathrm{X}$, Zhu B, Wang X, et al. (2016) Three-dimensional co-culture of mesenchymal stromal cells and differentiated osteoblasts on human bio-derived bone scaffolds supports active multi-lineage hematopoiesis in vitro: functional implication of the biomimetic HSC niche. Int J Mol Med 38, 1141-1151.

30. Abdallah BM (2017) Marrow adipocytes inhibit the differentiation of mesenchymal stem cells into osteoblasts via suppressing BMP-signaling. J Biomed Sci 24, 11.

31. Meuleman N, Tondreau T, Ahmad I, et al. (2009) Infusion of mesenchymal stromal cells can aid hematopoietic recovery following allogeneic hematopoietic stem cell myeloablative transplant: a pilot study. Stem Cells Dev 18, 1247-1252.

32. Bergstrom KS, Morampudi V, Chan JM, et al. (2015) Goblet cell derived RELM- $\beta$ recruits CD $4+\mathrm{T}$ cells during infectious colitis to promote protective intestinal epithelial cell proliferation. PLOS Pathog 11, e1005108.

33. Zanotti L, Angioni R, Calì B, et al. (2016) Mouse mesenchymal stem cells inhibit high endothelial cell activation and lymphocyte homing to lymph nodes by releasing TIMP-1. Leukemia 30, 1143-1154.

34. Boomsma RA \& Geenen DL (2012) Mesenchymal stem cells secrete multiple cytokines that promote angiogenesis and have contrasting effects on chemotaxis and apoptosis. PLOS ONE 7, e35685.

35. Zagoura DS, Roubelakis MG, Bitsika V, et al. (2012) Therapeutic potential of a distinct population of human amniotic fluid mesenchymal stem cells and their secreted molecules in mice with acute hepatic failure. Gut $\mathbf{6 1}$, 894-906.

36. Shibanuma M, Mashimo J, Mita A, et al. (1993) Cloning from a mouse osteoblastic cell line of a set of transforming-growthfactor-beta 1-regulated genes, one of which seems to encode a follistatin-related polypeptide. Eur J Biochem 217, 13-19.

37. Chaly Y, Marinov AD, Oxburgh L, et al. (2012) FSTL1 promotes arthritis in mice by enhancing inflammatory cytokine/ chemokine expression. Arthritis Rheum 64, 1082-1088.

38. Ballarò C, Ceccarelli S, Tiveron C, et al. (2005) Targeted expression of RALT in mouse skin inhibits epidermal growth factor receptor signalling and generates a Waved-like phenotype. EMBO Rep 6, 755-761.

39. Aqmasheh S, Shamsasanjan $\mathrm{K}$, Akbarzadehlaleh $\mathrm{P}$, et al. (2017) Effects of mesenchymal stem cell derivatives on hematopoiesis and hematopoietic stem cells. Adv Pharm Bull 7, 165-177.

40. Stagg J, Lejeune L, Paquin A, et al. (2004) Marrow stromal cells for interleukin-2 delivery in cancer immunotherapy. Hum Gene Ther 15, 597-608.

41. Nissen C, Moser Y, Weis J, et al. (1986) The release of interleukin-2 (IL-2) and colony stimulating activity (CSA) in aplastic anemia patients: opposite behaviour with improvement of bone marrow function. Blut 52, 221-230.

42. Yuan J, Huang G, Xiao Z, et al. (2013) Overexpression of $\beta$-NGF promotes differentiation of bone marrow mesenchymal stem cellsinto neurons through regulation of AKT and MAPK pathway. Mol Cell Biochem 383, 201-211.

43. Garcia-Lavandeira M, Quereda V, Flores I, et al. (2009) A GRFa2/Prop1/stem (GPS) cell niche in the pituitary. PLOS ONE 4, e4815.

44. Airaksinen MS \& Saarma M (2002) The GDNF family: signalling, biological functions and therapeutic value. Nat Rev Neurosci $\mathbf{3}$, 383-394

45. Li JP, Zheng CL \& Han ZC (2010) Abnormal immunity and stem/progenitor cells in acquired aplastic anemia. Crit Rev Oncol Hematol 75, 79-93. 